\title{
A Imagem Corporal de Mulheres Morbidamente Obesas Avaliada através do Desenho da Figura Humana
}

\author{
Graziela Aparecida Nogueira de Almeida ${ }^{12}$ \\ Sonia Regina Loureiro \\ José Ernesto dos Santos \\ Universidade de São Paulo, Ribeirão Preto
}

\begin{abstract}
Resumo
Objetiva-se avaliar a auto-imagem de 30 mulheres com obesidade mórbida, comparativamente a 30 mulheres não obesas. Procedeu-se à avaliação individual, utilizando-se o Desenho da Figura Humana (DFH) e entrevista complementar. Quanto aos aspectos gerais do desenho, os grupos diferiram significativamente em relação aos índices qualidade do grafismo, localização na página e temática, caracterizando predomínio de sentimentos de inadequação por parte das obesas. Quanto aos índices relativos ao tamanho do desenho, proporção, representação do tronco e tamanho de diferentes partes do corpo, os grupos diferiram entre si, sugerindo presença de indicadores de depreciação e distorção da imagem corporal entre as obesas. Observa-se que as mulheres obesas apresentaram dificuldade de expressar, simbolicamente, sua vivência corporal, sugerindo a presença de indicadores de sentimentos de inferioridade, descontentamento e preocupação com o corpo e a beleza.

Palavras-chave: Imagem corporal; desenho da figura humana; mulheres; obesidade.
\end{abstract}

The Body Image in Morbid Obese Women Evaluated through the Draw a Person Test

\begin{abstract}
The aim of this study was to assess self-image in 30 morbid obese women compared to 30 non obese women. Individual evaluation was carried out using the Draw a Person Test and a interview. Regarding general aspects of the drawing the groups differed significantly in quality of graphism, location in the page and theme indexes, indicating prevalence of feelings of enadequacy in the obese group. With regard to size of drawing, proportion, trunk representation and different body parts size indexes, the groups differed significantly, suggesting depreciation and distortion of body image in the obese group. It was observed that obese women had difficulty in expressing way their corporal existence, in a symbolic suggesting inferiority feelings, discontent and worry about body and beauty.

Keywords: Body image; draw a person test; women; obesity.
\end{abstract}

Durante muito tempo na história da humanidade, o ganho de peso, bem como o acúmulo de gordura, eram vistos como sinais de saúde e prosperidade. Hoje, contudo, a obesidade é considerada uma doença crônica, que afeta crianças, adolescentes e adultos, presente tanto em países desenvolvidos quanto em desenvolvimento (WHO, 1997).

De uma forma geral, a obesidade pode ser definida como uma doença resultante do acúmulo anormal ou excessivo de gordura sob a forma de tecido adiposo, de forma que possa resultar em prejuízos à saúde (Halpern, Matos, Suplicy, Mancini \& Zanella, 1998; WHO, 1997).

O estudo sobre os fatores etiológicos e mantenedores da obesidade deve ser multifatorial, envolvendo diferentes campos e abordagens como o físico, o psíquico, o ambiental e/ou genético (Eliaschewitz, Valente, Marquetti, Cohn \&

${ }^{1}$ Endereço para correspondência: Rua Adalberto Pajaúba, 957, B 11, $14055-$ 220, Ribeirão Preto, SP. Fone: (16) 63070020.E-mail: gnogueira@keynet.com.br ${ }^{2}$ Este trabalho foi realizado no Departamento de Clínica Médica, Ambulatório de Distúrbios de Conduta Alimentar do Hospital das Clínicas da Faculdade de Medicina de Ribeirão Preto, USP e no Centro Médico, Social e Comunitário de Vila Lobato, Ribeirão Preto, São Paulo.
Cukier, 1988; Friedman \& Brownell, 1995; Ingran, 1976; Pizzinatto, 1992). No que diz respeito à obesidade mórbida, Laurent e Vannotti (1993) afirmam que esta doença é o resultado de diversos obstáculos psicológicos - internos, relacionais, comportamentais e psicossociais. Nesse sentido, os aspectos psicossociais relacionados à obesidade têm sido estudados com ênfase diversa, incluindo avaliação da imagem corporal por meio de diferentes instrumentos, como desenhos, escalas e questionários.

No que diz respeito à avaliação por meio de desenhos, de acordo com Van Kolck (1984), o significado psicológico do desenho da figura humana tem suas bases no conceito de imagem corporal que, por sua vez, torna-se veículo de expressão da personalidade. Segundo a referida autora, a imagem corporal é projetada no desenho da figura humana e, conseqüentemente, reflete o conceito de si mesmo, além de expressar diferentes representações do indivíduo. Van Kolck, analisando as pesquisas sobre a catexe corporal definida como o grau de satisfação ou insatisfação com as várias partes e processo do corpo ou sentimentos da pessoa para com o seu corpo - aponta que esta tem sido relacionada 
ao autoconceito. Segundo a autora, os resultados destas pesquisas levaram às conclusões de que os sentimentos a respeito do corpo são proporcionais aos sentimentos sobre o eu.

Cash (1993) afirma que a imagem corporal refere-se à experiência psicológica de alguém sobre a aparência e o funcionamento do seu corpo. Segundo o autor, o descontentamento relacionado ao peso, que muitas vezes levam a uma imagem corporal negativa, advém de uma ênfase cultural na magreza e estigma social da obesidade.

De uma forma geral, os estudos sobre imagem corporal apontam para prejuízos relacionados à insatisfação, depreciação, distorção e preocupação com a auto-imagem, todos eles sendo fortemente influenciados por fatores sócio-culturais (Cash, 1993; Demarest \& Langer, 1996; Gittelson \& cols., 1996). Alguns autores, entre eles Foster, Wadden e Vogt (1997), Leonhard e Barry (1998) e Ogden e Evans (1996) fazem menção à Teoria do Descontentamento Normativo, que sustenta a idéia de que a insatisfação com o próprio corpo parece estar relacionada às exigências sociais e culturais de aparência e magreza.

Com base em revisão da literatura, Friedman e Brownell (1995) destacaram dois aspectos relativos à imagem corporal: insatisfação e distorção da imagem corporal. Quanto à insatisfação, os autores verificaram que ela parece estar associada à obesidade; enquanto que a distorção, embora associada à obesidade, os dados não têm se mostrado consistentes.

De acordo com os referidos autores, os aspectos físicos da obesidade têm sido documentados com detalhes, contudo os correlatos psicológicos do excesso de peso carecem ainda de mais estudos. Nesse contexto, entre as variáveis psicossociais que carecem de estudos, coloca-se a imagem corporal, bem como sua associação à obesidade.

Considerando-se a problemática descrita, o presente estudo objetivou caracterizar os aspectos psicossociais relativos à imagem corporal de mulheres com obesidade mórbida, comparativamente a um grupo de mulheres não obesas.

\section{Método}

\section{Participantes}

Foram estudados 60 sujeitos do sexo feminino, com idade variando de 30 a 60 anos, os quais foram divididos em dois grupos, de acordo com o Índice de Massa Corporal (IMC). O primeiro - Grupo Experimental (GE) - foi formado por pacientes que estavam em tratamento baseado em reeducação de hábitos alimentares e dieta, no Ambulatório de Distúrbios de Conduta Alimentar (ADCA) do Hospital das Clínicas da Faculdade de Medicina de Ribeirão Preto da Universidade de São Paulo, HCFMRP-USP, tendo diagnóstico médico de Obesidade Mórbida, definido a partir do critério de índice de massa corporal (IMC), que se baseia na correlação matemática Peso/Altura ${ }^{2}$. Neste caso, o IMC de todos os pacientes foi igual ou superior a $40 \mathrm{~kg} / \mathrm{m}^{2}$. O segundo - Grupo Controle (GC) - foi formado por mulheres com IMC variando de 20 a $25 \mathrm{~kg} / \mathrm{m}^{2}$, condição esta excludente para o diagnóstico de obesidade. Foram contatadas no Centro Médico, Social e Comunitário de Vila Lobato, em Ribeirão Preto, São Paulo, por ocasião de sua presença no serviço, buscando atendimento para si junto à Ginecologia, ou para seus filhos junto à Pediatria, ou ainda quando da participação em cursos profissionalizantes oferecidos à comunidade, quando então era solicitada a sua colaboração na pesquisa.

A caracterização dos sujeitos é apresentada na Tabela 1.

Observa-se que 50,0\% das mulheres morbidamente obesas e 53,3\% das mulheres não obesas apresentaram idade variando de 40 a 49 anos. A média de idade dos sujeitos obesos foi de 46,1 anos $(d p=5,95)$ e a dos não obesos foi de 42,6 anos $(d p=8,11)$. No que diz respeito ao IMC, todas

Tabela 1

Caracterização dos Sujeitos do GE Obesas (n=30) e do GC Não-obesas (n=30)

\begin{tabular}{|c|c|c|}
\hline \multirow[b]{2}{*}{ Características } & \multicolumn{2}{|c|}{$\%$} \\
\hline & GE obesas & GC não-obesas \\
\hline \multicolumn{3}{|l|}{ Idade } \\
\hline $30-39$ anos & 16,7 & 30,0 \\
\hline $40-49$ anos & 50,0 & 53,3 \\
\hline $50-60$ anos & 33,3 & 16,7 \\
\hline \multicolumn{3}{|c|}{ Índice de massa corporal (IMC) } \\
\hline$<25 \mathrm{~kg} / \mathrm{m}^{2}$ & - & 100,0 \\
\hline $40-49 \mathrm{~kg} / \mathrm{m}^{2}$ & 50,0 & - \\
\hline$\geq 50 \mathrm{~kg} / \mathrm{m}^{2}$ & 50,0 & - \\
\hline
\end{tabular}


as obesas apresentaram índices maiores ou iguais a $40 \mathrm{~kg} / \mathrm{m}^{2}$, enquanto que todas as não obesas apresentaram índices que variaram de 20 a $25 \mathrm{~kg} / \mathrm{m}^{2}$. O perfil descrito mostra que os sujeitos dos dois grupos são comparáveis em termos de idade e escolaridade e significativamente diferentes em termos de índice de massa corporal.

\section{Instrumentos}

Foram utilizados os seguintes instrumentos:

\section{Entrevista semi-estruturada}

Essa entrevista foi desenvolvida de acordo com os objetivos da pesquisa, como fonte de dados complementares, abordando tópicos relativos à profissão, peso e alimentação, situação clínica atual, relacionamento interpessoal e aspecto social. Para a finalidade desse estudo, serão considerados os aspectos relativos ao auto-relato relacionados à imagem corporal.

\section{Técnica do Desenho da Figura Humana}

Essa técnica foi aplicada e avaliada com base nas proposições de Van Kolck (1984), tendo por finalidade a avaliação da imagem corporal.

\section{Procedimento}

Os 30 sujeitos do GE foram contatados por carta ou durante a consulta médica. Os instrumentos foram aplicados individualmente pela primeira autora, em um consultório do ADCA do Hospital das Clínicas em condições ambientais adequadas.

Os 30 sujeitos do GC foram contatados no Centro Médico, Social e Comunitário de Vila Lobato. Os instrumentos foram aplicados individualmente pela primeira autora, em um consultório no próprio Centro Médico.

Com relação à técnica do Desenho da Figura Humana, foi solicitada a representação de duas figuras humanas completas. Assim, após a realização da primeira figura humana, era solicitado que representasse uma figura humana de outro sexo, diferente da primeira representada (Machover, 1949).

Os dados relativos à entrevista foram utilizados como fonte complementar de informações. Com relação ao Desenho da Figura Humana, os dados relativos às duas figuras humanas representadas foram cotejados individualmente conforme as recomendações de Van Kolck (1984), utilizando-se para tal um guia de avaliação aplicado às duas representações gráficas realizadas pelos 30 sujeitos de cada grupo. Visando a compreensão dos aspectos relacionados à imagem corporal, selecionou-se itens que, segundo a literatura, (Barros, Werutsky, Gütfriend, Biernat
\& Barros, 1990; Van Kolck, 1984) têm o seu significado diretamente associado à imagem corporal. Neste sentido foram selecionados itens relativos aos aspectos gerais, bem como ao tamanho e proporcionalidade dos desenhos.

Quanto aos aspectos gerais da representação gráfica, foram selecionados 10 itens, a saber: qualidade do grafismo, localização na página, temática, ordem de representação das figuras, tratamento diferencial das figuras, indicadores de conflito, articulações, linha mediana, rosto e roupas. Estes itens abrangem um total de 41 índices como possibilidade de codificação. Por exemplo, o item qualidade do grafismo abrangeu os seguintes índices: linha grossa, linha média, linha fina, traço contínuo, traço interrompido, traços de avanços e recuos e traço trêmulo.

Quanto aos aspectos relacionados ao tamanho e à proporcionalidade dos desenhos, foram selecionados 15 itens, a saber: tamanho em relação à folha, proporções e tamanho da cabeça, olhos, nariz, boca, pescoço, tronco, tórax ou peito, ombros, cintura, cadeiras e nádegas, braços, mãos e pernas. Estes itens abrangem um total de 53 índices como possibilidade de avaliação. Por exemplo, o item tamanho da cabeça abrangeu os seguintes índices: grande/ muito grande, média, pequena/muito pequena.

Participaram do procedimento de codificação das figuras humanas três psicólogos com experiência em avaliação psicológica, além da pesquisadora, distribuídos em duas duplas. Cada um dos protocolos, contendo os itens relativos às duas representações gráficas de cada sujeito, foi cotejado de forma independente por dois psicólogos. Quando da ocorrência de desacordo entre os dois avaliadores, a avaliação de consenso foi feita por um terceiro avaliador, participante da outra dupla de avaliadores. Visando avaliar o nível de concordância entre os dois avaliadores na classificação dos itens, procedeuse ao cálculo de acordo. Considerou-se para tal a fórmula de acordo simples, tomando-se como unidade de acordo a codificação de cada um dos itens do guia de avaliação.

Os resultados de acordo médio obtidos - 0,80 para o GE e 0,83 para o GC - com variação de 0,70 a 0,92 em ambos os grupos, podem ser considerados como aceitáveis.

Com o objetivo de comparar a produção dos grupos por meio do Desenho da Figura Humana, buscou-se descrever o perfil geral dos grupos com base nos índices predominantes, tomando-se como referência a freqüência e a porcentagem dos mesmos. Para uma comparação mais sistemática foram utilizados o Teste $\chi^{2}$ ou o Teste de Intervalo de Confiança entre Proporções. A escolha do teste deveu-se ao número de índices incluído em cada item. 
Tabela 2

Freqüência das Características Predominantes no Desenho da Figura Humana, com Base nos Índices Presentes na Produção Gráfica dos Participantes do GE Obesas (58 produções) e do GC Não-obesas (60 produções)

\begin{tabular}{|c|c|c|c|c|c|}
\hline \multirow[t]{2}{*}{ Itens/İndices } & \multicolumn{2}{|c|}{ GE Obesas } & \multicolumn{2}{|c|}{ GC Não-obesas } & \multirow{2}{*}{$\begin{array}{c}\text { Comparação } \\
\text { dos grupos }\end{array}$} \\
\hline & $F$ & $\%$ & $F$ & $\%$ & \\
\hline \multicolumn{6}{|l|}{ I- Aspectos gerais } \\
\hline \multicolumn{6}{|l|}{ Qualidade do grafismo } \\
\hline linha média & 33 & $56,90 \mathrm{a}$ & 42 & $70,00 \mathrm{a}$ & SD \\
\hline traço contínuo & 35 & $60,35 \mathrm{a}$ & 38 & $63,33 \mathrm{a}$ & SD \\
\hline \multicolumn{6}{|l|}{ Localização na folha } \\
\hline $4^{\circ}$ quadrante & 18 & 31,03 & 09 & 15,00 & $\mathrm{GE}>\mathrm{GC} *$ \\
\hline metade superior da folha & 12 & 20,69 & 24 & 40,00 & $\mathrm{GE}<\mathrm{GC} *$ \\
\hline \multicolumn{6}{|l|}{ Temática } \\
\hline figura mais jovem & 36 & $62,07 \mathrm{a}$ & 46 & $76,67 \mathrm{a}$ & SD \\
\hline \multicolumn{6}{|c|}{ Ordem de representação das figuras } \\
\hline sexo feminino em $1^{\circ}$ lugar & 20 & 68,97 & 21 & 70,00 & SD \\
\hline $\begin{array}{l}\text { Tratamento diferencial das fig } \\
\text { pouca diferenciação }\end{array}$ & \multicolumn{4}{|c|}{ Tratamento diferencial das figuras } & SD \\
\hline \multicolumn{6}{|l|}{ Indicadores de conflito } \\
\hline reforços & 32 & $55,17 \mathrm{a}$ & 36 & $60,00 \mathrm{a}$ & SD \\
\hline \multicolumn{6}{|l|}{ Articulações } \\
\hline $\begin{array}{l}\text { Linha mediana } \\
\text { presente }\end{array}$ & \multicolumn{4}{|c|}{ Linha mediana } & SD \\
\hline \multicolumn{6}{|l|}{ Rosto } \\
\hline representado & 28 & $48,28 \mathrm{a}$ & 25 & 41,67 & SD \\
\hline \multicolumn{6}{|l|}{ Roupas } \\
\hline traje comum completo & 47 & 81,03 & 40 & 66,67 & SD \\
\hline \multicolumn{6}{|c|}{ II- Tamanho e proporcionalidade } \\
\hline \multicolumn{6}{|c|}{ Tamanho em relação à folha } \\
\hline $\begin{array}{l}\text { Proporções } \\
\text { ausente }\end{array}$ & \multicolumn{4}{|c|}{ Proporções } & $\mathrm{GE}>\mathrm{GC} *$ \\
\hline \multicolumn{6}{|l|}{ Cabeça } \\
\hline grande/muito grande & 35 & 60,35 & 27 & 45,00 & $\mathrm{GE}>\mathrm{GC} *$ \\
\hline média/normal & 18 & 31,03 & 30 & 50,00 & $\mathrm{GE}<\mathrm{GC} *$ \\
\hline \multicolumn{6}{|l|}{ Olhos } \\
\hline pequenos & 33 & 56,90 & 11 & 18,33 & $\mathrm{GE}>\mathrm{GC} *$ \\
\hline médios & 21 & 36,21 & 38 & 63,33 & $\mathrm{GE}<\mathrm{GC} *$ \\
\hline \multicolumn{6}{|l|}{ Nariz } \\
\hline médio & 34 & 58,62 & 26 & 43,33 & SD \\
\hline \multicolumn{6}{|l|}{ Boca } \\
\hline média & 25 & 43,10 & 27 & 45,00 & SD \\
\hline \multicolumn{6}{|l|}{ Pescoço } \\
\hline comprido/longo & 34 & 58,62 & 27 & 45,00 & SD \\
\hline \multicolumn{6}{|l|}{ Tronco } \\
\hline distorção & 43 & 74,14 & 26 & 43,33 & $\mathrm{GE}>\mathrm{GC} *$ \\
\hline representação adequada & 15 & 25,86 & 31 & 51,67 & $\mathrm{GE}<\mathrm{GC} *$ \\
\hline Tórax ou peito & & & & & \\
\hline pequeno & 31 & 53,45 & 26 & 43,33 & SD \\
\hline Ombros & & & & & \\
\hline proporcionados & 24 & 41,38 & 28 & 46,67 & SD \\
\hline
\end{tabular}


Tabela 2

Freqüência das Características Predominantes no Desenho da Figura Humana, com Base nos Índices Presentes na Produção Gráfica dos Participantes do GE Obesas (58 produções) e do GC Não-obesas (60 produções)

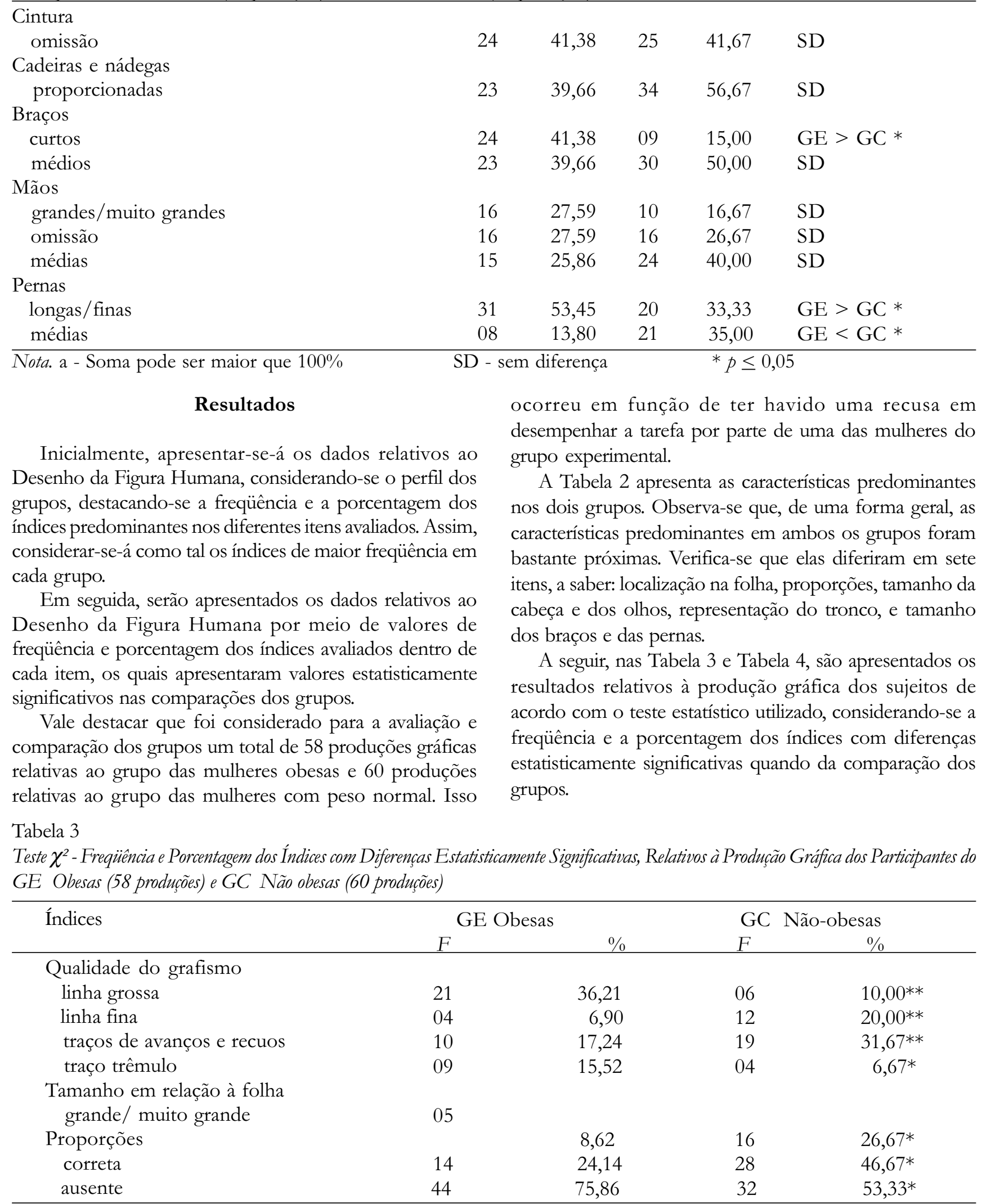

$* p<0,05 \quad * * p \leq 0,01$ 
Tabela 4

Teste de Intervalo de Confiança entre Proporções - Freqüência e Porcentagem dos Índices com Diferenças Estatisticamente Significativas, Relativos à Produção Gráfica dos Participantes do GE Obesas (58 produções) e GC Não-obesas (60 produções)

\begin{tabular}{|c|c|c|c|c|}
\hline \multirow[t]{2}{*}{ Índices } & \multicolumn{2}{|c|}{ GE Obesas } & \multicolumn{2}{|c|}{ GC Não-obesas } \\
\hline & $F$ & $\%$ & $F$ & $\%$ \\
\hline \multicolumn{5}{|l|}{ Localização na folha } \\
\hline $4^{\circ}$ Quadrante & 18 & 31,03 & 09 & $15,00^{*}$ \\
\hline metade superior & 12 & 20,69 & 24 & $40,00^{*}$ \\
\hline \multicolumn{5}{|l|}{ Temática } \\
\hline ausência de temática & 11 & 18,97 & 01 & $1,67 *$ \\
\hline \multicolumn{5}{|l|}{ Representação do tronco } \\
\hline distorção na forma & 43 & 74,14 & 26 & $43,33^{*}$ \\
\hline representação adequada & 15 & 25,86 & 31 & $51,67 *$ \\
\hline \multicolumn{5}{|l|}{ Tamanho do tórax ou peito } \\
\hline em ênfase ou grande & 11 & 18,97 & 02 & $3,33^{*}$ \\
\hline normal & 14 & 24,14 & 25 & $41,67 *$ \\
\hline omissão & 02 & 3,45 & 07 & $11,67^{*}$ \\
\hline \multicolumn{5}{|l|}{ Tamanho da cabeça } \\
\hline média/ normal & 18 & 31,03 & 30 & $50,00^{*}$ \\
\hline \multicolumn{5}{|l|}{ Tamanho dos olhos } \\
\hline grandes & 03 & 5,17 & 10 & $16,67^{*}$ \\
\hline médios & 21 & 36,21 & 38 & $63,33^{*}$ \\
\hline pequenos & 33 & 56,90 & 11 & $18,33^{*}$ \\
\hline \multicolumn{5}{|l|}{ Tamanho do nariz } \\
\hline grande & 04 & 6,90 & 14 & $23,33^{*}$ \\
\hline \multicolumn{5}{|l|}{ Tamanho do pescoço } \\
\hline médio & 06 & 10,35 & 14 & $23,33^{*}$ \\
\hline curto e grosso & 02 & 3,45 & 08 & $13,33^{*}$ \\
\hline \multicolumn{5}{|l|}{ Tamanho dos braços } \\
\hline curtos & 24 & 41,39 & 09 & $15,00^{*}$ \\
\hline \multicolumn{5}{|l|}{ Tamanho das pernas } \\
\hline longas/ longas e finas & 31 & 53,45 & 20 & $33,33^{*}$ \\
\hline médias & 08 & 13,80 & 21 & $35,00^{*}$ \\
\hline disparidade no tamanho & 07 & 12,07 & 01 & $1,67 *$ \\
\hline
\end{tabular}

$* p \leq 0,05$

Ao se tratar do item relativo à qualidade do grafismo, observa-se que as mulheres obesas, comparadas às mulheres com peso normal, apresentaram, em seus desenhos, freqüência significativamente maior de linhas grossas, bem como de traços trêmulos. Verifica-se, além disso, que as mulheres não obesas apresentaram em seus desenhos freqüência significativamente maior de linhas finas e traços caracterizados por avanços e recuos quando comparadas às obesas.

Quanto ao item relativo ao tamanho do desenho em relação à folha, observa-se que as mulheres não obesas se diferenciaram significativamente das obesas, apresentando mais desenhos de tamanho grande ou muito grande.
Ao se tratar das proporções entre as várias partes do corpo desenhado, nota-se que as mulheres obesas fizeram mais desenhos desproporcionais do que as não obesas.

Quanto à localização dos desenhos, pode-se observar que as obesas tiveram mais desenhos localizados no $4^{\circ}$ quadrante do que as mulheres não obesas. Os grupos também se diferenciaram significativamente quanto à localização dos desenhos na metade superior da folha, com o grupo das mulheres com peso normal tendo apresentado mais desenhos com tal localização.

No que diz respeito à temática, nota-se que o grupo das mulheres obesas apresentou mais desenhos sem um tema específico do que as com peso normal. 
Os grupos também se diferenciaram significativamente no que diz respeito à representação do tronco, onde se observa que as obesas apresentaram, em seus desenhos, formas mais distorcidas do que as mulheres com peso normal.

Observa-se ainda que, ao se tratar da representação do tórax ou peito, as mulheres morbidamente obesas desenharam com freqüência significativamente maior o tórax ou peito de forma enfatizada ou de tamanho grande comparativamente às não obesas, as quais apresentaram o tórax ou peito com tamanho normal ou o omitiram.

No item relativo ao tamanho da cabeça, observa-se, no grupo das mulheres não obesas, freqüência significativamente maior de cabeças representadas com o tamanho normal.

Quanto à representação dos olhos, nota-se que as mulheres obesas desenharam com freqüência significativamente maior os olhos de tamanho pequeno, enquanto que as mulheres com peso normal fizeram mais representações de olhos com tamanhos normais ou grandes.

Ao se tratar do item que avalia o tamanho do nariz, o grupo das mulheres não obesas representou nariz de tamanho grande com freqüência significativamente maior do que as obesas.

$\mathrm{Na}$ representação do pescoço, pode-se observar que o grupo das não obesas representou o pescoço de tamanhos médio e curto e grosso com freqüência significativamente maior do que as mulheres obesas.

No que diz respeito ao tamanho dos braços, observase que as mulheres morbidamente obesas representaram os braços de tamanho curtos com freqüência significativamente maior do que as não obesas.

Com relação à representação das pernas, nota-se que as mulheres obesas representaram as pernas longas/ longas e finas e com disparidade no tamanho das mesmas, com freqüência maior do que as mulheres com peso normal, as quais representaram pernas de tamanho médio.

É importante salientar que todos os itens foram tratados estatisticamente. Porém, os itens que se seguem - ordem de representação das figuras, tratamento diferencial das figuras, diferenciação acentuada das figuras, indicadores de conflitos, articulações, linha mediana, rosto, roupas, bem como tamanho da boca, dos ombros, da cintura, de cadeiras e nádegas e das mãos - não apresentaram diferença significativa na comparação dos grupos.

\section{Discussão}

O valor diagnóstico do Desenho da Figura Humana tem sido objeto de questionamentos e polêmica quanto à validade preditiva da técnica. Entre outros autores em nosso meio, Hutz e Bandeira (1994) discutem tais questões enfocando a subjetividade envolvida no julgamento dos indicadores gráficos. A necessidade de estudos sistemáticos com o Desenho da Figura Humana, que avaliem a qualidade psicométrica da técnica e seus parâmetros de avaliação, há muito se faz presente. Os estudos de grupos específicos de sujeitos não respondem integralmente tal questão, mas, na medida em que respeitam formas sistemáticas de coleta e análise dos indicadores gráficos, podem contribuir para dar suporte científico à ampla utilização clínica dos desenhos.

Considerando-se os resultados obtidos por meio do Desenho da Figura Humana, a discussão dos mesmos estará fundamentada nas atribuições de significados aos índices com base nas proposições de Van Kolck (1984), e na integração destes com dados obtidos por meio das entrevistas.

No que diz respeito ao perfil geral dos dois grupos, os dados apontam para um número bastante elevado de itens onde as características predominantes se mostraram as mesmas. No entanto, os itens que apresentaram diferenças quanto às características predominantes foram aqueles que parecem ter um significado clínico bastante relevante no que diz respeito à questão da imagem corporal. No grupo das mulheres morbidamente obesas, o predomínio de desenhos localizados no $4^{\circ}$ quadrante da folha, de cabeça de tamanho grande ou muito grande, de olhos pequenos, do tronco representado de forma distorcida, de mãos de tamanhos grandes, muito grandes ou de sua omissão, bem como de pernas representadas como longas e finas, sugere alguns indicadores de prejuízos da auto-imagem. De uma forma geral, esses dados apontam sentimentos de subestima e de descontentamento com relação ao próprio corpo, além de uma falta de confiança em si e nos relacionamentos interpessoais. Parece importante salientar que, nos itens onde as características predominantes foram diferentes nos dois grupos, o que se observa é que, no grupo das mulheres com peso normal, as características predominantes apontam para sinais comuns, os quais dispensam interpretação já que não constituíam objeto de análise.

Quando os grupos foram estatisticamente comparados, foi possível observar que, ao se tratar dos aspectos gerais referentes à representação gráfica dos sujeitos não obesos, a freqüência acentuada de linhas finas, de traços caracterizados por avanços e recuos e de desenhos localizados na metade superior da folha sugere manifestações de insegurança e de dificuldade de adaptação ao ambiente por parte dessas mulheres. As representações gráficas das mulheres obesas, caracterizadas por linhas grossas, traços trêmulos, maior número de desenhos localizados no quarto quadrante da 
folha e falta de uma temática específica, pode estar relacionada a manifestações de insegurança, ansiedade, bem como dificuldade de adaptação ao ambiente.

A ausência de temática, particularmente, parece ter relação com uma possível dificuldade da mulher obesa em expressar de forma simbólica a sua imagem corporal. Também pode estar associada a sentimentos de inferioridade, inadequação e depreciação diante do risco de julgamentos externos negativos.

Os dados referentes à entrevista também descrevem auto-relatos de sentimentos de inadequação e insatisfação com a auto-imagem por parte das mulheres obesas. Quanto às entrevistas, elas relataram, por exemplo, que não saem de casa porque sentem vergonha de serem gordas. É importante ressaltar que tais conseqüências negativas foram sempre atribuídas ao excesso de peso corporal. Esses sentimentos parecem estar diretamente relacionados com a ênfase que, atualmente, tem sido dada aos corpos magros, os quais têm se tornado sinônimos de beleza, normalidade e saúde, em especial entre as mulheres (Cash, 1993; Foster \& cols., 1997; Leonhard \& Barry, 1998; Ogden \& Evans, 1996).

Quanto aos dados relativos ao tamanho e proporcionalidade dos desenhos, observa-se, entre as mulheres não obesas, a presença de sinais de agressividade, de dependência e de falta de controle para com o ambiente, o que pode ser percebido por meio de desenhos representados em tamanhos grandes, bem como por olhos e nariz também de tamanhos grandes. O pescoço, representado por meio de tamanho médio, curto e grosso, parece um indicador sugestivo de que essas mulheres são guiadas mais pelos impulsos do que pela reflexão. Elas parecem confiar nas suas funções intelectuais e sociais, o que pode ser percebido por meio da representação da cabeça de tamanho normal. A representação do tronco e do tórax ou peito de forma adequada, além das pernas de tamanhos normais sugere que essas mulheres estão satisfeitas com sua forma corporal, além de apresentarem uma estabilidade com relação ao corpo. Em oposição, no grupo das mulheres morbidamente obesas, a presença significativa de desenhos sem proporção entre as várias partes do corpo pode estar associada a uma dificuldade em perceber o seu corpo como de fato ele é, o que pode ser considerado um indicador sugestivo de distorção da imagem corporal. A representação do tronco de forma distorcida e do tórax ou peito em ênfase ou de tamanho grande pode estar associada a um descontentamento dessas mulheres com o próprio corpo, somada a uma preocupação com a beleza corporal.

Por meio da forma como as mulheres obesas representaram os braços, os olhos e as pernas, pode-se detectar indicadores sugestivos de falta de confiança na própria produtividade bem como na manipulação do ambiente, além de uma inadequação no contato com as pessoas, somada a uma dificuldade de inserção no ambiente. Estes indicadores têm também seu significado associado à necessidade de autonomia, o que se contrapõe à presença de sentimentos ambivalentes, estes relacionados ao fato de se considerarem inadequadas para alcançar a sua independência pessoal.

Analisando-se em conjunto os resultados obtidos por meio da técnica gráfica, é possível destacar, no grupo das mulheres obesas, a relação proposta por Van Kolck (1984) e Ogden e Evans (1996) entre grau de satisfação com a imagem corporal e o auto-conceito. Segundo os referidos autores, os sentimentos que uma pessoa tem sobre o seu próprio corpo são proporcionais aos sentimentos que nutre sobre ela própria. No presente estudo, as representações gráficas das obesas sugerem a presença de indicadores de comprometimento da imagem corporal, bem como indicadores de auto-conceito negativo, estes associados à presença de sinais de ansiedade, insegurança e sentimentos de inadequação. Nesse caso, a insatisfação corporal pode estar associada às verbalizações sugestivas de auto-conceito negativo por parte das mulheres obesas.

Entre as mulheres com peso normal não se observa, por meio da técnica gráfica, indicadores de comprometimento da imagem corporal. Ao contrário, parecem satisfeitas com o seu corpo. No entanto, observase, por meio da entrevista, que algumas delas relataram se sentirem insatisfeitas com o corpo atual, já que verbalizaram se sentirem gordas, apesar do IMC normal.

Esses dados são concordantes com as observações de Sarwer, Wadden e Foster (1998), que sugerem que a percepção subjetiva que uma pessoa tem sobre seu corpo pode ser mais importante do que a realidade objetiva de sua aparência. Nesse sentido, o peso, por si só, não parece ser um único determinante do grau de satisfação ou insatisfação com a imagem corporal. Os dados também são concordantes com os achados de Leonhard e Barry (1998) que verificaram que mulheres com peso normal se sentiam gordas, independente do seu peso. Segundo os autores, isso pode ser interpretado como um indício de confusão que algumas pessoas apresentam sobre o tamanho do próprio corpo em relação às expectativas da sociedade. Mais uma vez, parece que as normas sociais de magreza exercem uma forte influência sobre a percepção da imagem corporal. O estudo de Ogden e Evans (1996) parece um importante referencial neste contexto. Os referidos autores constataram que, ao ser apontado para os sujeitos que seu peso estava abaixo do normal, estes mostravam uma melhora significativa no humor. Os autores argumentam que, de acordo com a 
normas sociais de atratividade, possivelmente estar abaixo do peso parece desejável para as mulheres em geral, além de reforçar o seu auto-conceito positivo.

Parece interessante ressaltar aqui que, por meio da técnica gráfica e da entrevista, foi possível notar que, de uma forma geral, as mulheres com peso normal pareciam confiar nas suas funções sociais. Neste caso, parece haver uma relação direta entre confiança social e relato de autopercepção positiva da imagem corporal dessas mulheres. Ao se tratar das mulheres obesas, esta relação também parece se fazer presente, porém, em um sentido contrário. Ou seja, a insegurança e os sentimentos de inadequação das mesmas frente ao ambiente parecem estar associados ao seu baixo auto-conceito. Concordando com Demarest e Langer (1996), a auto-percepção da imagem corporal parece depender, em parte, do status do indivíduo diante das normas sociais e culturais aceitas.

Os sinais de comprometimento da imagem corporal presentes entre as obesas parecem estar relacionados à insatisfação, depreciação, preocupação e a sinais de distorção da imagem corporal.

Os dados aqui encontrados sinalizam-se também concordantes com achados da literatura envolvendo a investigação da imagem corporal de pessoas obesas, a saber, os estudos de Barros e colaboradores (1990), Cash (1993), Demarest e Langer (1996), Ogden e Evans (1996) e Werlinger, King, Clark, Pera e Wincze (1997). Esses autores constataram a presença de indicadores de insatisfação e preocupação com o corpo associados a diferentes variáveis, como a inaceitação do próprio corpo, os valores sócioeconômico-culturais e o status do sujeito. Com a finalidade de tentar compreender a presença de tais indicadores de insatisfação e preocupação com a imagem corporal entre as obesas, parece importante recorrer à Teoria do Descontentamento Normativo, citada por Ogden e Evans (1996), Foster e colaboradores (1997) e Leonhard e Barry (1998). Considerando que a magreza tem se tornado um imperativo social e cultural, como sustenta a referida teoria, pode-se supor que as mulheres com obesidade mórbida que participaram desse estudo possam estar sendo influenciadas por tais imposições. Brodie, Drew e Jackman (1996) verificaram que as pessoas parecem ter uma tendência esteriotipada de avaliar a imagem corporal em função de normas sociais e culturais de peso saudável. Como conseqüência, a insatisfação com o próprio corpo, o qual é bastante diferente dos padrões aceitáveis de peso, bem como a preocupação com o "ser/estar diferente", passam a se fazer presentes na vida dessas pessoas que, por fim, sofrem ainda mais pela sua obesidade.

A presença de indicadores de comprometimento da imagem corporal entre as obesas pode, ainda, estar relacionada ao fato de que elas estavam sob tratamento médico baseado em reeducação de hábitos alimentares e dieta. Tal suposição está fundamentada nas considerações de Cash (1993), Ben-Tovin e Walker (1994) e Werlinger e colaboradores (1997) que afirmam que pessoas obesas que buscam tratamento para perda de peso são diferentes dos obesos da população geral e podem estar mais preocupadas com a sua imagem corporal. Segundo Cash (1993), pessoas obesas que se inscrevem em programas para perda de peso, em geral, apresentam uma imagem corporal negativa, bem como um forte investimento na sua aparência. Nota-se, por meio dos relatos na entrevista, que as mulheres obesas parecem estar incomodadas e insatisfeitas com o seu corpo. Parece existir um desejo de melhorar a aparência, que seria alcançada, segundo a percepção das mulheres obesas, através da perda de peso.

Os sinais de distorção da imagem corporal encontrados nesse estudo podem estar associados a uma dificuldade da mulher obesa em aceitar o seu corpo como ele realmente é. Levando-se em consideração a descrição de imagem corporal proposta por Slade (1994), como uma ilustração do tamanho, imagem e forma do corpo e os sentimentos associados a tais características e às diferentes partes do corpo, pode-se pensar, com base nos indicadores presentes na produção gráfica, que as obesas construíram uma idéia pouco realística acerca do seu corpo atual. A representação feita pelas obesas reflete a expressão de seus desejos ou aspirações, ou ainda uma projeção de uma imagem ideal.

Provavelmente as mulheres experimentam a pressão de fatores internos e externos para tal idealização. Quanto aos fatores externos, pode-se destacar a cultura de valorização dos corpos magros, que parece impelir as mulheres a desejarem perder peso e, assim, adequarem-se aos padrões de beleza. Quanto aos fatores internos, possivelmente os prejuízos experimentados quanto à saúde física também podem estar levando à busca de perda de peso. Nesse sentido, observa-se que, por razões internas ou externas, as mulheres obesas, insatisfeitas com o seu corpo atual, buscam alternativas para a perda de peso, como o programa de reeducação alimentar, no qual estão se tratando. Neste caso, a dieta, bem como as demandas de ajustes e de autocontrole, parecem ser novas fontes de sofrimento emocional em função das restrições impostas.

Percebe-se, assim, diversas fontes para os sentimentos de inadequação presentes nos indicadores da técnica gráfica relativos à imagem corporal de mulheres morbidamente obesas, abrangendo uma diversidade de aspectos. Nesse sentido, considera-se importante a avaliação da imagem corporal enquanto um recurso para a compreensão de aspectos psicológicos relativos à obesidade. 


\section{Referências}

Barros, C. A. S. M., Werutsky, C. A., Gütfriend, C., Biernat, E. S. \& Barros, T. M. (1990). Transtorno da imagem corporal de obesos em grupoterapia. Revista de Psiquiatria do Rio Grande do Sul, 12(2), 75-83.

Ben-Tovin, D. I. \& Walker, M. K. (1994). The influence of age and weight on women's body attitudes as measured by the body attitudes questionnaire (BAQ). Journal of Psychosomatic Research, 38(5), 477-481.

Brodie, D. A., Drew, S. C. \& Jackman, C. (1996). Influence of preconception on body image. Perceptual and Motor Skills, 83(2), 571-577.

Cash, T. F. (1993). Body-image attitudes among obese enrollees in a commercial weight-loss program. Perceptual and Motor Skills, 77(3), 10991103.

Demarest, J. \& Langer, E. (1996). Perception of body shape by underweight, average, and overweight men and women. Perceptual and Motor Skills, 83(2), 569-570.

Eliaschewitz, F. G., Valente, O., Marquetti, L. C., Cohn, K. G. \& Cukier, R. (1988). Obesidade. Ars. Curandi., 21(1), 16-17, 20-22.

Foster, G. D., Wadden, T. A. \& Vogt, R. A. (1997). Body image in obese before, during, and after weight loss treatment. Health Psychology, 16(3), 226-229.

Friedman, M. A. \& Brownell, K. D. (1995). Psychological correlats of obesity: Moving to the next research generation. Psychological Bulletin, 117(1), 3-20.

Gittelson, J., Harris, S. B., Thorne-Lyman, A. L., Hanley, A. J. G., Barnie, A. \& Zinman, B. (1996). Body image concepts differ by age and sex in an Ojibway-Cree community in Canada. Journal of Nutrition, 126, 29903000 .

Halpern, A., Matos, A. F. G., Suplicy, H. L., Mancini, M. C. \& Zanella, M. T. (1998). Obesidade. São Paulo: Lemos.

Hutz, C. S. \& Bandeira, D. R. (1994, Outubro). Avaliação psicológica com o Desenho da Figura Humana: Técnica ou intuição? Trabalho apresentado na XXIV Reunião Anual de Psicologia. Ribeirão Preto, SBP.
Ingram, D. H. (1976). Psychoanalytic treatment of the obese person: I. American Journal of Psychoanalysis, 36(1), 35-41.

Laurent, J. A. \& Vannotti, M. (1993). Handcaps of massive obesity. Rev. Prat., 43(15), 1908-1910.

Leonhard, M. L. \& Barry, N. J. (1998). Body image and obesity: Effects of gender and weight on perceptual measures of body image. Addictive Behaviors, 23(1), 31-34.

Machover, K. (1949). Proyección de la personalidad en el dibujo de la figura bumana. Habana: Cuba Cultural.

Ogden, J. \& Evans, C. (1996). The problem with weighing: Effects on mood, self-esteem and body image. International Journal of Obesity and Related Metabolic Disoders, 20(3), 272-277.

Pizzinatto, V. T. (1992). Obesidade infantil. São Paulo: Sarvier.

Sarwer, D. B., Wadden, T. A. \& Foster, G. D. (1998). Assessment of body image dissatisfaction in obese women: Specificity, severity, and clinical significance. Journal of Consulting and Clinical Psychology, 66(4), 651-654.

Slade, P. D. (1994). What is body image? Behavioral Research Theraphy, 32(5), 497-502.

Van Kolck, O. L. (1984). Testes projetivos gráficos no diagnóstico psicológico. São Paulo: EPU.

Werlinger, K., King, T. K., Clark, M. M., Pera, V. \& Wincze, J. P. (1997). Perceived changes in sexual functioning and body image following weight loss in an obese female population: A pilot study. Journal of Sex \& Marital Therapy, 23 (1), 74-78.

World Health Organization - WHO (1997). Obesity: Preventing and managing the global epidemic. Geneva.

\section{Sobre os autores}

Graziela Aparecida Nogueira de Almeida é Psicóloga, Mestre em Ciências Médicas pela Faculdade de Medicina de Ribeirão Preto da Universidade de São Paulo e Doutoranda em Psicologia pela Faculdade de Filosofia, Ciências e Letras de Ribeirão Preto, Universidade de São Paulo.

Sonia Regina Loureiro é Psicóloga, Docente do Departamento de Neurologia, Psiquiatria e Psicologia Médica da Faculdade de Medicina de Ribeirão Preto, Universidade de São Paulo.

José Ernesto dos Santos é Médico, Docente na Universidade de São Paulo- Ribeirão Preto e é responsável pelo Ambulatório de Distúrbios de Conduta Alimentar do Hospital de Clínicas da Faculdade de Medicina de Ribeirão Preto. 